Expert Group, Diemen/Oegstgeest, The Netherlands and Department of Clinical and Health Psychology, Utrecht University; Trudy Mooren is a clinical psychologist and senior researcher, F. Jackie June ter Heide is a clinical psychologist and $\mathrm{PhD}$ student and Niels van der $\mathbf{A a}$ is a senior researcher and methodologist, all with Arq Psychotrauma Expert Group, Diemen/Oegstgeest.

\section{References}

1 American Psychiatric Association. Diagnostic and Statistical Manual of Mental Disorders, Fourth Edition, Text Revision (DSM-IV-TR). APA, 2000.

2 Fazel $M$, Jeremy $W$, Danesh J. Prevalence of serious mental disorder in 7000 refugees resettled in western countries: a systematic review. Lancet 2005; 365: 130914

3 Porter M, Haslam N. Predisplacement and postdisplacement factors associated with mental health of refugees and internally displaced persons: a meta-analysis. JAMA 2005; 294: 60212.

4 Lindert J, von Ehrenstein OSV, Priebe S, Mielck A, Brähler E. Depression and anxiety in labor migrants and refugees a systematic review and meta-analysis. Soc Sci Med 2009; 69: 24657.

5 Steel Z, Chey T, Silove D, Marnane C, Bryant RA, Van Ommeren M Association of torture and other potentially traumatic events with mental health outcomes among populations exposed to mass conflict and displacement: a systematic review and meta-analysis. JAMA 2009; 302: 53749.
6 Momartin S, Silove D, Manicavasagar V, Steel Z. Comorbidity of PTSD and depression: associations with trauma exposure, symptom severity and functional impairment in Bosnian refugees resettled in Australia. J Affect Disord 2004; 80: 2318.

7 Nickerson A, Liddell BJ, Maccallum F, Steel Z, Silove D, Bryant RA. Posttraumatic stress disorder and prolonged grief in refugees exposed to trauma and loss. BMC Psychiatry 2014; 14: 106.

8 Mollica RF, Caspi-Yavin Y, Bollini P, Truong T, Tor S, Lavelle J. The Harvard Trauma Questionnaire: validating a cross-cultural instrument for measuring torture, trauma, and posttraumatic stress disorder in Indochinese refugees. J Nerv Ment Dis 1992; 180: 1116.

9 Mollica RF, Wyshak G, De Marnette T, Tu B, Yang T, Khuon F, et al Hopkins Symptom Checklist (HSCL-25): Manual for Cambodian, Laotian and Vietnamese versions. Torture 1996; 6: 3542

10 Hollifield M, Warner TD, Lian N, Krakow B, Jenkins JH, Kesler J, et al. Measuring trauma and health status in refugees: a critical review. JAMA 2002; 288: 61121.

11 Stevens JP. Applied Multivariate Statistics for the Social Sciences. Erlbaum, 2002

12 Johnson $\mathrm{H}$, Thompson A. The development and maintenance of post traumatic stress disorder (PTSD) in civilian adult survivors of war trauma and torture: a review. Clin Psychol Rev 2008; 28: 3647.

\title{
Difficult to treat? A comparison of the effectiveness of treatment as usual in refugees and non-refugees
}

\author{
F. Jackie June ter Heide, ${ }^{1}$ Geert E. Smid ${ }^{1}$
}

BJPsych Bulletin (2015), 39, 182-186, doi: 10.1192/pb.bp.114.047928

${ }^{1}$ Foundation Centrum '45/partner in Arq, The Netherlands

Correspondence to Jackie June ter Heide (j.ter.heide@centrum45.nl)

First received 30 Apr 2014, final revision 20 Feb 2015, accepted 12 Mar 2015

(C) 2015 The Authors. This is an openaccess article published by the Royal College of Psychiatrists and distributed under the terms of the Creative Commons Attribution License (http:// creativecommons.org/licenses/by/ 4.0), which permits unrestricted use, distribution, and reproduction in any medium, provided the original work is properly cited.
Aims and method To examine treatment response in traumatised refugees, we compared routine outcome monitoring data (Harvard Trauma Questionnaire) of two refugee populations with those of individuals experiencing profession-related trauma who were treated at a specialised psychotrauma institute.

Results Asylum seekers/temporary refugees $(n=21)$ and resettled refugees $(n=169)$ showed significantly lower post-traumatic stress disorder (PTSD) symptom reduction between intake and 1 year after intake than did a comparison group of non-refugees $(n=37)$, but the interaction effect was clinically small (partial $\eta^{2}=0.03$ ). Refugees who had more severe symptoms at intake showed significantly greater symptom reduction after 1 year.

Clinical implications Therapists and refugee patients should have realistic expectations about response to treatment as usual. Additional treatment focusing on improving quality of life may be needed for refugees whose PTSD symptom severity remains high. At the same time, novel approaches may be developed to boost treatment response in refugee patients with low responsiveness.

Declaration of interest None.
Among many clinicians, traumatised asylum seekers and refugees have a reputation of being difficult to treat. Low treatment response in refugees is often attributed to patient-related factors, ${ }^{1}$ such as trauma history, current stressors and complex psychopathology. Many asylum seekers and refugees have been exposed to multiple, prolonged, interpersonal traumatic events such as war and human trafficking. ${ }^{2}$ In addition, they have to handle the 
stress of forced migration, including involvement in legal procedures $^{3}$ and loss of their home country, cultural resources, family and social status. ${ }^{4}$ Apart from posttraumatic stress disorder (PTSD), ${ }^{5}$ they may experience comorbid symptoms including depression, anxiety and psychosis, ${ }^{6}$ as well as symptoms sometimes referred to as complex PTSD. ${ }^{7}$ By contrast, some clinicians argue that it is the treatment offered to refugees, rather than their potential to benefit from treatment, that leads to low treatment response, ${ }^{8}$ and that refugees, like other adults with chronic PTSD, ${ }^{9}$ should be treated with trauma-focused interventions. One way to examine treatment response in refugees is by comparing the effectiveness of different kinds of treatment in refugee samples. In recent years, randomised trials have shown promising effects for trauma-focused treatment in refugees. ${ }^{10}$ Another way to examine treatment response is to compare the effectiveness of treatment in refugee samples and non-refugee samples. This has been done little, if at all. This study's aim is to compare traumatised asylum seekers' and refugees' response to treatment as usual with that of another multiply traumatised population: indivduals affected by profession-related trauma (i.e. military veterans and police officers).

\section{Method}

\section{Setting}

Data were collected in Foundation Centrum '45, a Dutch mental health institute specialising in treatment of complex psychotrauma. Specific populations include asylum seekers and refugees, veterans of various peace missions, World War II resistance fighters as well as concentration camp survivors and their offspring, and police officers. Centrum ' 45 receives national referrals of patients who, owing to their psychosocial complexity, cannot be treated in general mental healthcare or who have shown insufficient response to treatment in general mental healthcare. Treatment for PTSD (individually or in groups) generally consists of a combination of supportive therapy, pharmacotherapy and trauma-focused therapy, particularly eye movement desensitisation and reprocessing (EMDR), ${ }^{11}$ narrative exposure therapy (NET) ${ }^{12}$ and brief eclectic psychotherapy for PTSD (BEPP). ${ }^{13}$ As these three trauma-focused treatments are evidence based, choice of treatment mainly depends on the therapist's training. Art therapy, psychomotor therapy and music therapy are also offered, especially to patients who follow a clinical or day-clinical programme.

\section{Assessments}

To evaluate the effectiveness of treatment as usual, Centrum '45 has routinely administered assessments at intake and at the end of treatment. Since 2007, a routine outcome monitoring (ROM) assessment 1 year after intake has been added for all patient populations. Since its introduction, ROM response has increased from around 40 to $55 \%$ in 2012 . For several years, the Harvard Trauma Questionnaire (HTQ) ${ }^{14}$ was used as a ROM instrument with refugees and for a shorter period also with non-refugee populations. The HTQ has been specifically designed for use with refugee populations. It is a self-report instrument that consists of two parts: one focusing on traumatic events and one on symptoms of post-traumatic stress (specific to DSM-IV ${ }^{15}$ and additional symptoms reported by traumatised refugees). Symptoms are rated on a four-point scale ranging from 1 (not at all) to 4 (extremely). A mean score of 2.5 has been recommended as cut-off score for PTSD, ${ }^{16}$ although this recommendation has not been validated in a wide range of patient populations.

\section{Sample}

To answer our research question, we had at our disposal a ROM data-set that consisted of 577 patients who had completed assessments both at intake and 1 year after intake (with a range of 8 to 16 months). From this data-set, we excluded all partners and children of war-affected persons ( $n=218$; primarily children of parents traumatised in World War II) because their reasons for seeking help, generally speaking, do not include PTSD. We then excluded all patients who at intake had not been administered the HTQ $(n=125)$ but another instrument to assess PTSD (a Dutch self-rating inventory for PTSD). ${ }^{16}$ As the final dataset contained only a small number of patients traumatised during World War II $(n=7)$, we also excluded those patients. The final data-set consisted of 227 patients who had had their second assessment between March 2007 and April 2013. We divided the sample into three groups: asylum seekers/temporary refugees (i.e. those who are still awaiting the decision on their asylum application and those who have obtained temporary refugee status, which may not be extended after 5 years), resettled refugees (i.e. those who have obtained permanent refugee status or subsequent Dutch nationality), and patients with profession-related trauma (i.e. military veterans and police officers).

\section{Statistical analysis}

All analyses were performed using SPSS version 20.0 for Windows. Demographical and clinical characteristics were calculated, and chi-squared and $t$-tests were conducted to check for demographical and clinical differences between the groups. For the HTQ, mean PTSD severity at intake (T1) and one year after intake (T2) was computed as well as the difference between the two (PTSD symptom reduction). We checked HTQ variables for extreme outliers, but we found none. Missing data for the HTQ consisted of missing mean scores at T2 for 7 patients (2 asylum seekers/temporary refugees and 5 resettled refugees) and missing events scores at $\mathrm{T} 1$ for 42 patients (4 asylum seekers/temporary refugees, 34 resettled refugees and 4 professionals). We handled missing data by using pair-wise deletion.

We conducted pair-wise $t$-tests to determine treatment response within each group, and calculated by hand the effect sizes $\left(\eta^{2}\right)$. Following Cohen, we interpreted $\eta^{2}=0.01$ to be a small effect, $\eta^{2}=0.06$ as moderate and $\eta^{2}=0.14$ as large. ${ }^{17}$ We set confidence intervals at $95 \%$. To examine potential differences in treatment response between the three groups, we conducted repeated measures analysis of variance (ANOVA), using time as within-subjects factor and group as between-subjects factor. For the interaction effect, an effect-size (partial $\eta^{2}$ ) of 0.01 was interpreted to be small,

\section{Bulletin}




\begin{tabular}{|c|c|c|c|c|c|c|}
\hline & $\begin{array}{l}\text { Asylum seekers/ } \\
\text { temporary } \\
\text { refugees }(n=21)\end{array}$ & $\begin{array}{l}\text { Resettled } \\
\text { refugees } \\
(n=169)\end{array}$ & $\begin{array}{l}\text { Profession- } \\
\text { related trauma } \\
\quad(n=37)\end{array}$ & $F$ & d.f. & $P$ \\
\hline \multicolumn{7}{|l|}{ Demographic characteristics } \\
\hline Age, years: mean (s.d.) & $36.1(10.4)$ & $43.8(8.9)$ & $44.5(8.6)$ & 7.32 & 2 & 0.001 \\
\hline Male, $n(\%)$ & $12(57.1)$ & $123(72.8)$ & $34(91.9)$ & & 2 & $0.009^{a}$ \\
\hline \multicolumn{7}{|l|}{ Clinical characteristics } \\
\hline HTQ score at intake, mean (s.d.) & $3.14(0.35)$ & $3.08(0.52)$ & $2.80(0.53)$ & 6.72 & 2 & 0.002 \\
\hline Traumatic event types (HTQ), ${ }^{b}$ : mean (s.d.) & $13.9(4.0)$ & $12.3(5.4)$ & $9.3(4.6)$ & 8.12 & 2 & 0.001 \\
\hline Time between assessments, months: mean (s.d.) & $12.1(1.7)$ & $12.1(1.4)$ & $10.6(1.5)$ & 16.09 & 2 & $<0.001$ \\
\hline
\end{tabular}

HTQ, Harvard Trauma Questionnaire.

a. $\chi^{2}=9.47$.

b. Experienced or witnessed

0.09 as medium and 0.25 as large. ${ }^{17}$ To examine variables associated with treatment response in asylum seekers and refugees, we performed a multiple regression analysis with PTSD symptom reduction (HTQ score at T1 minus HTQ score at T2) as the dependent variable and demographic variables (gender, age and refugee status (no/temporary/ permanent)) and clinical variables (PTSD severity at T1, number of traumatic event types and time between assessments) as independent variables.

\section{Results}

\section{Demographic characteristics}

For demographic and clinical characteristics of the final sample, see Table 1.

Because this study was observational, we found significant differences in demographic and clinical characteristics between the three groups for all variables. Asylum seekers/temporary refugees came predominantly from Afghanistan, Armenia, Iraq and Sierra Leone $(n=3$, $14.3 \%$ for each country); resettled refugees came predominantly from the former Yugoslavia $(n=59,34.9 \%)$, Iraq $(n=28,16.6 \%)$ and Afghanistan $(n=22,13.0 \%)$; and patients who had profession-related trauma came predominantly from The Netherlands $(n=33,89.2 \%)$. The traumatic events that the asylum seekers/temporary refugees most frequently reported were physical torture $(n=17,81.0 \%)$, threat of physical torture $(n=17,81.0 \%)$ and other life-threatening situation $(n=17,81.0 \%)$. Resettled refugees most frequently reported being close to death $(n=135,79.9 \%)$, other life-threatening situation $(n=125$, $74.0 \%)$ and forced isolation from family $(n=123,72.8 \%)$. Professionals most frequently reported other lifethreatening situation $(n=34,91.9 \%)$, combat situation $(n=34,91.9 \%)$, serious injury $(n=31,83.8 \%)$ and being close to death $(n=31,83.8 \%)$.

\section{Treatment outcome}

Figure 1 shows the results of the repeated measures ANOVA for the three groups.

Mean PTSD symptom severity decreased from 3.13 (95\% CI 2.91 to 3.35 ) to 2.92 (95\% CI 2.65 to 3.20 ) for asylum seekers/temporary refugees; from 3.10 (95\% CI 3.03 to 3.18 ) to 2.88 (95\% CI 2.79 to 2.98) for resettled refugees; and from 2.80 (95\% CI 2.64 to 2.96 ) to 2.31 (95\% CI 2.11 to 2.51 ) for

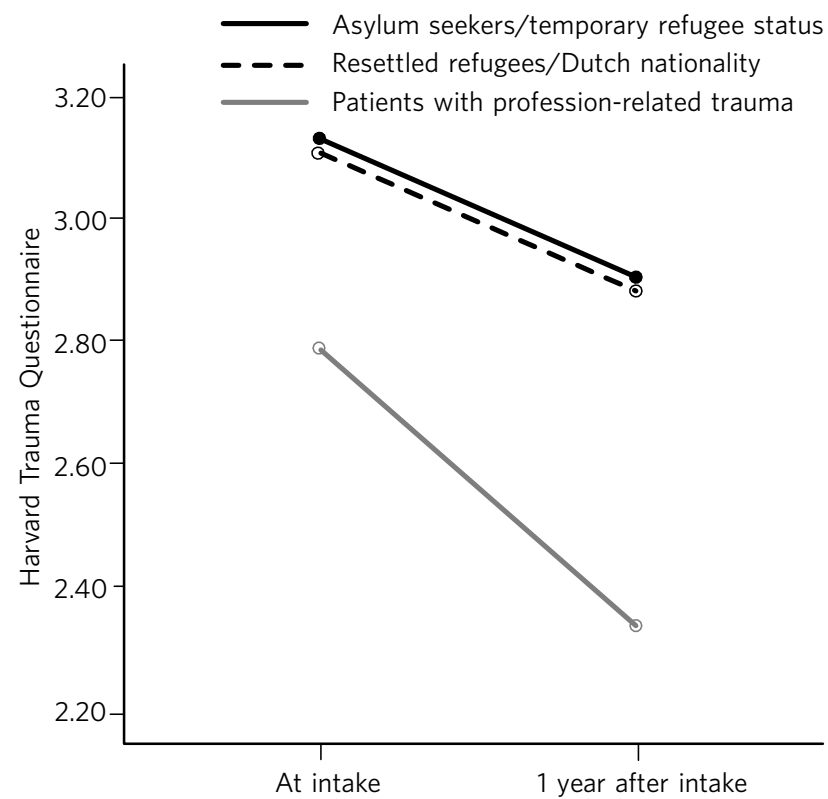

Fig. 1 PTSD symptom severity at intake and after 1 year.

patients suffering from profession-related trauma. Pairedsamples $t$-tests revealed a significant decrease in PTSD severity for resettled refugees $\left(0.22\right.$, s.d. $=0.52, t_{(163)}=5.39$, $P<0.001)$ and for professionals $\left(0.49\right.$, s.d. $=0.64, t_{(36)}=4.65$, $P<0.001)$, but not for the smallest group, asylum seekers/ temporary refugees $\left(0.21\right.$, s.d. $\left.=0.59, t_{(18)}=1.53, P=0.143\right)$. Effect sizes for treatment response in asylum seekers/ temporary refugees and resettled refugees were moderate $\left(\eta^{2}=0.12\right.$ and $\eta^{2}=0.15$, respectively); effect size for patients with profession-related trauma was large $\left(\eta^{2}=0.38\right){ }^{16}$ Repeated measures ANOVA showed a significant effect for time $(F=32.27, P<0.001)$ with a medium effect size (partial $\eta^{2}=0.13$ ), and a significant group $\times$ time interaction effect $(F=3.65, \quad P=0.028)$ with a small effect size (partial $\left.\eta^{2}=0.03\right)^{16}$

We then combined the two refugee groups and, using multiple regression analysis, we examined whether seven demographic and clinical variables were associated with PTSD symptom reduction (Table 2).

As shown in Table 2, refugee patients with more severe PTSD symptoms at intake had significantly stronger reductions in PTSD symptom severity after 1 year. The other variables were not significantly associated with PTSD 


\begin{tabular}{|c|c|c|c|c|}
\hline & B & $95 \% \mathrm{Cl}$ & $\beta$ & $P$ \\
\hline \multicolumn{5}{|l|}{ Demographic variables } \\
\hline Gender & 0.11 & -0.07 to 0.29 & 0.09 & 0.238 \\
\hline Age & 0.00 & -0.01 to 0.00 & -0.09 & 0.277 \\
\hline \multicolumn{5}{|l|}{ Refugee status } \\
\hline None $v$. permanent & -0.03 & -0.37 to 0.31 & -0.01 & 0.872 \\
\hline Temporary $v$. permanent & -0.14 & -0.52 to 0.24 & -0.06 & 0.464 \\
\hline \multicolumn{5}{|l|}{ Clinical variables } \\
\hline PTSD symptom severity at intake (HTQ) & 0.48 & 0.32 to 0.64 & 0.45 & $<0.001$ \\
\hline Traumatic event types (HTQ), $n$ & -0.01 & -0.03 to 0.00 & -0.11 & 0.157 \\
\hline Time between assessments, months & -0.01 & -0.06 to 0.05 & -0.01 & 0.851 \\
\hline
\end{tabular}

B, regression coefficient; $\beta$, standardised regression coefficient; HTQ, Harvard Trauma Questionnaire; PTSD, post-traumatic stress disorder

symptom reduction. The percentage of variance explained by the model $\left(\mathrm{R}^{2}\right)$ was $21.5 \%$.

\section{Discussion}

This study shows that asylum seekers/temporary refugees and resettled refugees experienced significantly lower PTSD symptom reduction between intake and 1 year after intake than did a comparison group of multiply traumatised military veterans and police officers. However, greatest differences between groups were found in PTSD symptom severity at intake and 1 year after intake rather than in PTSD symptom reduction. Explorations of PTSD symptom reduction in refugees showed that those who had more severe symptoms at intake experienced significantly greater symptom reduction after 1 year; other variables (including variables related to refugee status and number of traumatic events) were not related to symptom reduction.

The results show that despite specialised treatment being offered to refugees, treatment response can be limited and PTSD severity frequently remains high. Possible explanations, and consequently clinical implications, might be threefold: patient-related, therapist-related and treatment-related. As for patient-related factors, the multiple determinants of PTSD might influence refugees' ability to benefit from treatment. It is generally acknowledged that PTSD in refugees is influenced by both traumatic and current stressors, some (or many) of which may be beyond the patients' and therapists' control. ${ }^{18}$ Following this explanation, clinicians and patients should have realistic expectations about what treatment may achieve in such a heavily traumatised and burdened population. Interventions that focus on improving quality of life rather than on further symptom reduction, such as acceptance and commitment therapy, ${ }^{19}$ might be useful for those patients who despite prolonged treatment continue to suffer from clinically significant PTSD. Clinicians sometimes suspect asylum seekers to exaggerate symptoms to remain in medical care and thereby increase the chance of obtaining a refugee status. We found no substantiation for this hypothesis of 'secondary gain' - in our study, not having a permanent refugee status was not associated with a decreased treatment response.

As for therapist-related factors, therapeutic skills that might suffice in trauma-focused treatment of other multiply traumatised groups might fall short in the treatment of refugees. Therapists might need more extensive training and supervision regarding choosing and staying with a treatment focus, categorising and selecting of target memories, and understanding and restructuring of trauma-related cognitions in order not to lose their way in the multitude of symptoms, memories and transcultural challenges. At the same time, therapists need to maintain a sense of being 'good enough' to provide treatment to refugees with limited responsiveness. ${ }^{20}$

Finally, regarding treatment-related factors, not all evidence-based treatments will work with all refugees. Therapists will need to explore non-response, and they may need to consult refugee patient populations themselves $^{21}$ to examine which treatment aims and techniques speak to refugees who insufficiently benefit from treatment as usual. In addition, novel approaches may be developed to enhance treatment response. Centrum ' 45 is currently exploring the feasibility of refugee treatment that focuses primarily on prolonged grief rather than on PTSD, and of intranasal oxytocin as a novel strategy to boost treatment response in refugees. ${ }^{22}$

\section{Limitations}

Although this study is valuable for comparing the effects of treatment as usual in refugee populations with those in a non-refugee population (which, to our knowledge, has not been done before), it also has several limitations. First, a division of the asylum seeker group into asylum seekers and temporary refugees, and of the profession-related trauma group into military veterans and police officers, would have been clinically meaningful but was not possible due to limited sample sizes for these groups. Second, some variables that might have shed light on differences in treatment response between the three groups (including comorbid disorders, the amount and content of treatment, change in refugee status and chronicity of PTSD) were not included in the data-set. Future studies should use a broader range of variables to more comprehensively assess predictors of refugees' treatment responses. Third, ROM assessments at our institute are completed by about $55 \%$ of patients, and findings might not generalise to our complete patient population, nor to traumatised refugees in general.

Nevertheless, our study contributes to the debate on refugees' treatment response by showing that it is indeed 
relatively lower than that of multiply traumatised nonrefugees.

\section{Acknowledgement}

We thank Niels van der Aa for providing us with the data-set and methodological information.

\section{About the authors}

F. Jackie June ter Heide MA, MPhil (Cantab) is a clinical psychologist/ psychotherapist and researcher, Foundation Centrum '45/partner in Arq, Oegstgeest and Diemen, The Netherlands, and Geert E. Smid MD, PhD is a psychiatrist and senior researcher, Foundation Centrum '45/partner in Arq.

\section{References}

1 Carlsson JM, Mortensen EL, Kastrup M. A follow-up study of mental health and health-related quality of life in tortured refugees in multidisciplinary treatment. J Nerv Ment Dis 2004; 193: 651-7.

2 Silove D, Tarn R, Bowles R, Reid J. Psychosocial needs of torture survivors. Aust NZ J Psychiat 1991; 25: 481-90.

3 Hodes M, Goldberg D. The treatment of refugees: service provision reflects Britain's ambivalence. Psychiatr Bull 2002; 26: 1-2.

4 Summerfield D. Asylum-seekers, refugees and mental health services in the UK. Psychiatr Bull 2001; 25: 161-3.

5 American Psychiatric Association. Diagnostic and Statistical Manual of Mental Disorders, 5th Edition (DSM-5). APP, 2013.

6 Fazel M, Wheeler J, Danesh J. Prevalence of serious mental disorder in 7000 refugees resettled in western countries: a systematic review. Lancet 2005; 365: 1309-14.

7 Lab D, Santos I, De Zulueta F. Treating post-traumatic stress disorder in the 'real world': evaluation of a specialist trauma service and adaptations to standard treatment approaches. Psychiatr Bull 2008; 32: $8-12$.

8 Başoğlu M. Rehabilitation of traumatised refugees and survivors of torture: after almost two decades we are still not using evidence based treatments. BMJ 2006; 333: 1230-1.
9 National Institute for Clinical Excellence. Post-Traumatic Stress Disorder: The Management of PTSD in Adults and Children in Primary and Secondary Care. Gaskell, 2005.

10 Nickerson A, Bryant RA, Silove D, Steel Z. A critical review of psychological treatments of posttraumatic stress disorder in refugees. Clin Psychol Rev 2011; 31: 399-417.

11 ter Heide FJJ, Mooren TM, Kleijn W, De Jongh A, Kleber RJ. EMDR versus stabilisation in traumatised asylum seekers and refugees: results of a pilot study. Eur J Psychotraumatol 2011; 2: 5881.

12 Stenmark H, Catani C, Neuner F, Elbert T, Holen A. Treating PTSD in refugees and asylum seekers within the general health care system: a randomized controlled multicentre study. Behav Res Ther 2013; 51: 641-7.

13 Nijdam MJ, Gersons BPR, Reitsma JB, de Jongh A, Olff M. Brief eclectic psychotherapy $\mathrm{V}$. eye movement desensitisation and reprocessing therapy for post-traumatic stress disorder: randomised controlled trial. Br J Psychiatry 2012; 200: 224-31.

14 Mollica RF, Caspi-Yavin Y, Lavelle J, Tor S, Yang T, Chan S, et al. The Harvard trauma questionnaire (HTQ): manual; Cambodian, Laotian and Vietnamese versions. Torture 1996; 6 (suppl 1): 19-34.

15 American Psychiatric Association. Diagnostic and Statistical Manual of Mental Disorders (4th edn). APA, 1994

16 Hovens JE, Bramsen I, Van der Ploeg HM. Self-rating inventory for posttraumatic stress disorder: review of the psychometric properties of a new brief Dutch screening instrument. Percept Mot Skills 2002; 94: 996-1008.

17 Cohen J. Statistical Power Analysis for the Behavioral Sciences. Erlbaum, 1988.

18 Miller KE, Rasmussen A. War exposure, daily stressors, and mental health in conflict and post-conflict settings: bridging the divide between trauma-focused and psychosocial frameworks. So Sci Med 2010; 70 7-16.

19 Welch S, Rothbaum BO. Emerging treatments for PTSD. In Handbook of PTSD: Science and Practice (ed M Friedman, TM Keane, PA Resick): 469-96. Guilford Press, 2007.

20 Linehan MM. Cognitive-Behavioral Treatment of Borderline Personality Disorder. Guilford Press, 1993.

21 Murphy D, Ndegwa D, Kanani A, Rojas-Jaimes C, Webster A. Mental health of refugees in inner-London. Psychiatr Bull 2002; 26: 222-4.

22 Olff $M$, Langeland $W$, Witteveen A, Denys D. A psychobiological rationale for oxytocin in the treatment of posttraumatic stress disorder. CNS Spect 2010; 15: 522-30. 